\title{
Hard x-ray transmission curved crystal spectrometers (10-100 keV) for laser fusion experiments at the ShenGuang-III laser facility
}

\author{
Ming-hai Yu ${ }^{1,2}$, Guang-yue $\mathrm{Hu}^{1}$, Ning $\mathrm{An}^{3}$, Feng Qian ${ }^{2}$, Yu-chi $\mathrm{Wu}^{2}$, Xiao-ding Zhang ${ }^{1}$, Yu-qiu Gu ${ }^{2}$, \\ Qiu-ping Wang ${ }^{3}$, and Jian Zheng ${ }^{1}$ \\ ${ }^{1}$ Department of Modern Physics \& CAS Key Laboratory of Geospace Environment, University of Science and Technology of China, \\ Hefei, Anhui 230026, China \\ ${ }^{2}$ Research Center of Laser Fusion, China Academy of Engineering Physics, Mianyang 621900, China \\ ${ }^{3}$ National Synchrotron Radiation Laboratory, University of Science and Technology of China, Hefei, Anhui 230026, China \\ (Received 14 August 2015; revised 8 October 2015; accepted 21 December 2015)
}

\begin{abstract}
Two transmission curved crystal spectrometers are designed to measure the hard x-ray emission in the laser fusion experiment of Compton radiography of implosion target on ShenGuang-III laser facility in China. Cylindrically curved $\alpha$-quartz (10-11) crystals with curvature radii of 150 and $300 \mathrm{~mm}$ are used to cover spectral ranges of 10-56 and 17-100 keV, respectively. The distance between the crystal and the x-ray source can be changed over a broad distance from 200 to $1500 \mathrm{~mm}$. The optical design, including the integral reflectivity of the curved crystal, the sensitivity, and the spectral resolution of the spectrometers, is discussed. We also provide mechanic design details and experimental results using a Mo anode x-ray source. High-quality spectra were obtained. We confirmed that the spectral resolution can be improved by increasing the working distance, which is the distance between the recording medium and the Rowland circle.
\end{abstract}

Keywords: curved crystal spectrometer; hard x-ray; laser fusion

\section{Introduction}

Hard x-ray emission with $E>10 \mathrm{keV}$ plays an important role in laser-driven inertial confinement fusion. When the laser or laser-produced soft x-rays compress the deuterium and tritium (DT) capsule, energetic electrons generated during the laser-plasma interaction preheat the DT fuel and thus disturb a low-entropy implosion. By measuring the emitted hard x-rays, the number of the energetic electrons can be quantified and the preheat levels inferred ${ }^{[1,2]}$. Core hard $\mathrm{x}$-ray emission of implosion targets can provide a signature of the mixing ${ }^{[3]}$, symmetry ${ }^{[4,5]}$, temperature, and density conditions of the fuel capsule ${ }^{[6,7]}$ near its peak compression. X-rays of imploding targets can also be an efficient hard $x$-ray source for high-energy radiography of dense or high- $Z$ targets $^{[8,9]}$. For Compton radiography of implosion targets ${ }^{[10]}$, a hard $\mathrm{x}$-ray backlighter source is generated by intense short pulses laser irradiation on a target,

Correspondence to: G.-y. Hu. Department of Modern Physics, University of Science and Technology of China, No 96, Jinzhai Road, Hefei, Anhui Province, China. Email: gyhu@ ustc.edu.cn which is then transmitted through the implosion target to determine the area density and symmetry of the fuel capsule near peak compression. Precise diagnosis of the hard X-ray spectrum of the backlighter source, and the core radiation of the implosion target which induces the background, is crucial to obtain a sufficiently high signal over background for Compton radiography of implosion targets.

Solid-state detectors of single-photon counting CCDs (3-30 keV ${ }^{[11]}$, dispersive $\mathrm{x}$-ray detectors of transmission curved crystal spectrometers $(10-120 \mathrm{keV})^{[12]}$, and nondispersive X-ray detectors with filters, such as Ross pairs spectrometers $(18-90 \mathrm{keV})^{[13,14]}$, filter stack spectrometers $(12-700 \mathrm{keV})^{[15,16]}$ and filter-fluorescer spectrometers (20$220 \mathrm{keV})^{[17,18]}$ are the conventional approaches for hard $\mathrm{x}$-ray spectra diagnostics. The dispersive $\mathrm{x}$-ray detectors of transmission curved crystal spectrometers are superior, especially in the 10-120 keV range which is more applicable to laser fusion experiments, due to their high spectral resolution, broad energy coverage, and robustness in harsh environments. Transmission curved crystal spectrometers have been commissioned on almost all of the laser fusion 
facilities such as Omega ${ }^{[19-21]}$ and $\mathrm{NIF}^{[22]}$. They were also utilized on many short pulse laser facilities ${ }^{[23,24]}$ and a fast Z-pinch facility ${ }^{[25]}$ for experiments of $\mathrm{x}$-ray source, atomic physics, medical radiography, etc.

Experiments of Compton radiography of implosion targets are planned at the ShenGuang-III (SG-III) laser facility (200 kJ/3 ns/351 nm/48 beams) in China, which has begun running recently, to obtain spatial profiles of the fuel capsule area density of fuel capsule near peak compression. Core hard x-ray emission of implosion targets, hard x-ray emission induced by energetic electrons on gold hohlraum and hard x-ray backlighter sources produced by short pulse PW laser beams are the three crucial components of the Compton radiography experiments. Prior to the actual Compton radiography experiment, we designed two transmission curved crystal spectrometers to detect the hard x-ray signal and record spectra in the $10-100 \mathrm{keV}$ range. These spectrometers employed two cylindrically curved $\alpha$-quartz (10-11) crystals with curvature radii of 150 and $300 \mathrm{~mm}$ to cover the 10-56 and 17-100 keV photon energy ranges, respectively. The details of the optical and mechanical designs, and preliminary experimental results employing a Mo anode X-ray source, are presented.

\section{Optical design and theoretic calculations}

\subsection{Optical design}

The optical design of the spectrometer adopts the curved crystal optics proposed by Cauchois ${ }^{[26]}$. As shown in Figure 1, X-rays emitted from the X-ray source are diffracted by the curved crystal. After passing through a polychromatic crossover point on the central optical axis, the dispersed $\mathrm{x}$ rays are recorded by a planar detector perpendicular to the central optical axis of the crystal. The diffraction angle obeys the Bragg's law,

$$
2 d \sin \theta=n \lambda,
$$

where $d$ is the lattice constant of the crystal's diffraction planes, $\theta$ is the diffraction angle, $n$ is the diffraction order, $\lambda=h c / E$ represents the wavelength of the $\mathrm{x}$-ray with photon energy $E, h$ is the Plank constant, $c$ is the light velocity in vacuum.

Thus, x-rays with lower energies have larger diffraction angles and are dispersed to further lateral distances from the central optical axis. The lateral distance can be calculated by

$$
y=R \sin \theta / \cos \beta+d_{0} \tan \beta,
$$

where $y$ is the lateral distance on the detecting surface from the central optical axis of the dispersed $\mathrm{x}$-ray, $\beta$ is the angle between the dispersed x-ray and the crystal's optical axis which is defined as $\beta=2 \theta-\sin ^{-1}[R \sin \theta /(R+s)], s$ is the distance from the $\mathrm{x}$-ray source to the crystal (detecting

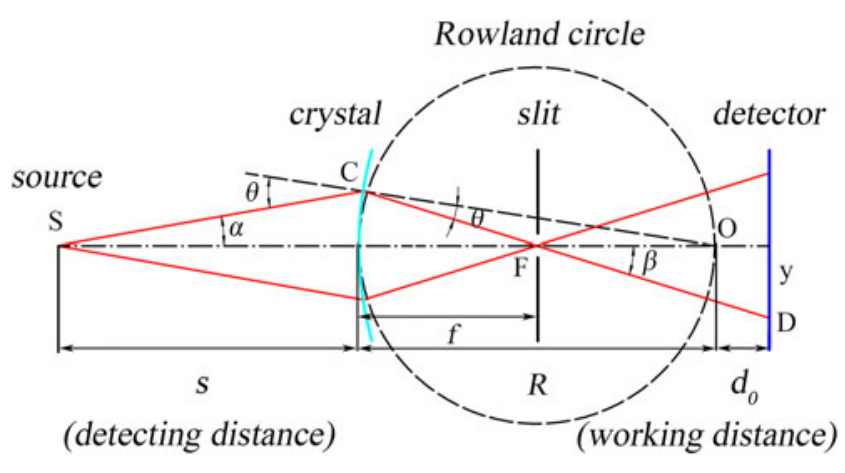

Figure 1. Cauchois-geometry optics of a symmetry transmission cylindrical curved crystal.

distance), $R$ is the curvature radius of the cylindrically curved crystal, $d_{0}$ is the distance from detecting surface to the Rowland circle (working distance).

The relationship between the lateral distance of the dispersed spectral line and its photon energy ${ }^{[19]}$, referred to as the 'plate function', is not easy to obtain by directly solving Eq. (2) ${ }^{[27]}$. The plate function is usually obtained by fitting the measured spectrum with the form in ${ }^{[12]}$

$$
E^{2}=a+b\left(y-y_{0}\right)^{-2}+c\left(y-y_{0}\right)^{2},
$$

where $a, b, c$ and $y_{0}$ are fitting coefficients.

A diffracted x-ray with the same photon energy will pass through a crossover point on the central optical axis. In order to improve the signal-to-noise ratio of the recording medium, a slit is usually placed here to absorb the x-rays that transmit straight through the crystal without diffraction. The position of the slit depends on the x-ray photon energy and the distance between the x-ray source and the crystal (the detecting distance), which can be calculated by

$$
f=R(1-\sin \theta / \sin \beta)
$$

where $f$ is the distance between the slit (crossover point) and the crystal.

Figure 2 shows the relations of the position of the slit versus the detecting distance of the Mo K $\alpha_{1}$ line $(17.48 \mathrm{keV})$, and the $\mathrm{x}$-ray photon energy with the detecting distance fixed at $500 \mathrm{~mm}$. The curved crystals' curvature radii are 150 and $300 \mathrm{~mm}$. The data in the figure indicate that the position of the slit is sensitive to the detecting distance but not sensitive to the x-ray photon energy. Thus, the position of the slit should be adjusted when the detecting distance is changed.

To cover the low energy limit of $10 \mathrm{keV}$, we calculated the lateral distance of the footprint (Point $\mathrm{C}$ in Figure 1) of the $10 \mathrm{keV}$ x-ray on the crystal, which are shown in Figure 3. The lateral distance increases with the crystal's curvature radius and the detecting distance. The effective diffraction length of the $\alpha$-quartz crystal we can obtain is about $55 \mathrm{~mm}$. So we chose the curved crystal's curvature 


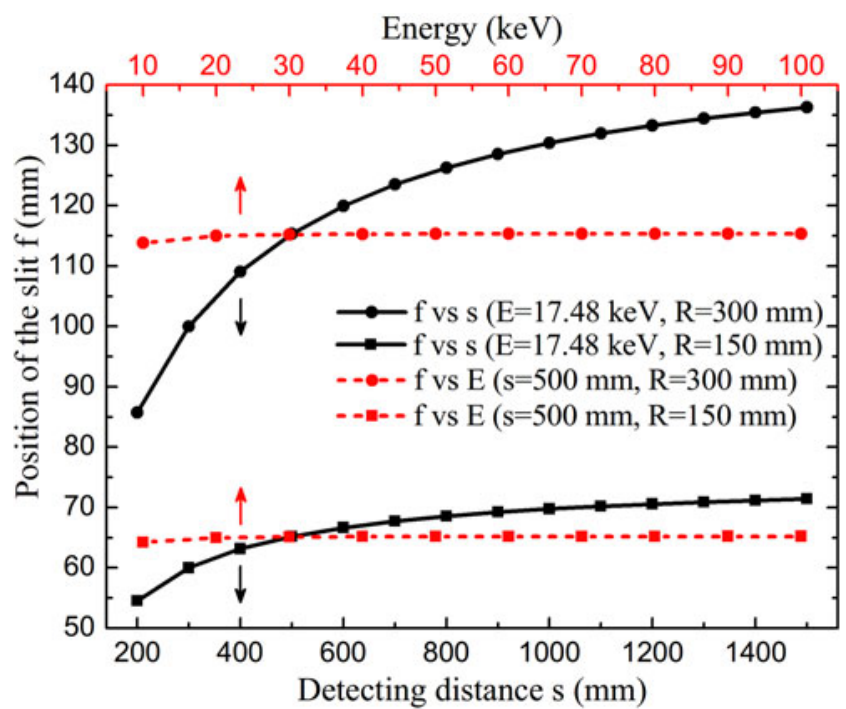

Figure 2. (Solid lines) the position of the slit $f$ versus the detecting distance $s$ (the source-to-crystal distance) of the $17.48 \mathrm{keV}$ Mo K $\alpha_{1} \mathrm{x}$-ray. (Dash lines) the position of the slit $f$ versus the x-ray photon energy at a fixed detecting distance, $s$, of $500 \mathrm{~mm}$. The crystals' curvature radii are 150 and $300 \mathrm{~mm}$.

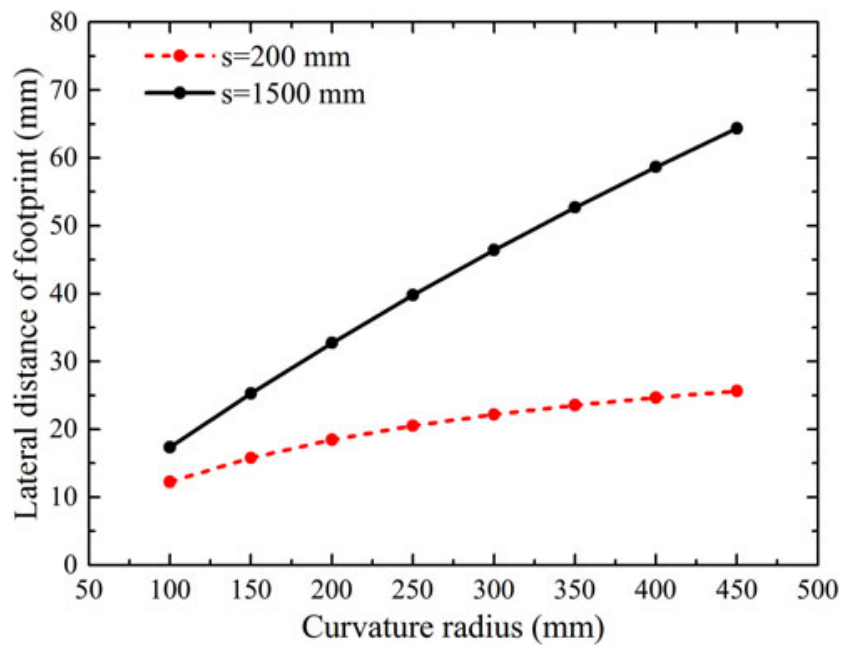

Figure 3. The lateral distance of the $10 \mathrm{keV} x$-ray footprint on the crystal (Point $\mathrm{C}$ in Figure 1) versus the crystal's curvature radius. The detecting distances $s$ are $200 \mathrm{~mm}$ (dash line) and $1500 \mathrm{~mm}$ (solid line).

radius to be $150 \mathrm{~mm}$ in the Low Energy Curved Crystal Spectrometer (LCCS) to cover the low-energy range of 10 $56 \mathrm{keV}$. However, curved crystal with smaller curvature radius of $150 \mathrm{~mm}$ has low spectral resolution at higherenergy ranges of $>30 \mathrm{keV}$ (see Figure 4). To obtain high resolution in the high-energy range, we add another High Energy Curved Crystal Spectrometer (HCCS) with a crystal curvature radius of $300 \mathrm{~mm}$ to cover the energy range of 17 $100 \mathrm{keV}$.

Design parameter details for these two spectrometers are shown in Table 1. Thin $\alpha$-quartz (10-11) slices with $70 \mathrm{~mm}$ length, $12 \mathrm{~mm}$ width, and $0.27 \mathrm{~mm}$ thickness were chosen

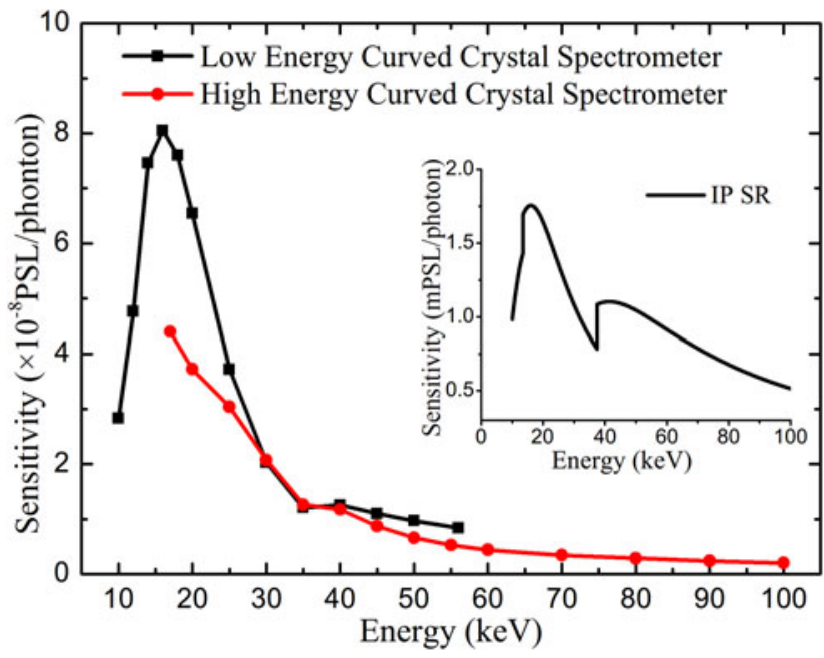

Figure 4. Theoretical spectral sensitivities of the transmission curved crystal spectrometers LCCS and HCCS. Insert is the sensitivity curve of Fujifilm ${ }^{\mathrm{TM}}$ image plates SR.

to fabricate the curved crystal due to the material's high reflectivity, small Darwin width, flexibility, and long-term stability. The lattice constant of the (10-11) crystallographic plane is $3.343 \AA$. The curvature radii of the curved crystals are $150 \mathrm{~mm}$ for LCCS and $300 \mathrm{~mm}$ for HCCS. Image plates (IPs, Fujifilm BAS-SR) are used to record the dispersed xrays at the detecting surface.

\subsection{Sensitivity of the spectrometers}

The reflectivity of the curved crystal, which is the intensity ratio of the diffracted $\mathrm{x}$-rays to the incident $\mathrm{x}$-rays, can be calculated using the Takagi-Taupin equations ${ }^{[28]}$. We used the XOP code ${ }^{[29]}$, which numerically solves the TakagiTaupin equations, to calculate the theoretical reflectivity of the curved crystal in our design. As shown in Figure 5, the energy-dependent integrated reflectivity of the curved crystals decreases with the curvature radius. This is consistent with the lamellar model ${ }^{[30]}$ which treats the curved crystal as a stack of very thin flat crystals (lamellar) slightly misaligned with each other. The decreased curvature radius will increase the misalignment between lamellae. Thus, the Darwin width of the reflectivity curve will broaden, resulting in increases in the integrated reflectivity, although the peak of the reflectivity curve will decrease a little. However, increasing the Darwin width also causes a decrease in spectrometer resolution (see Figure 4).

Fujifilm $^{\mathrm{TM}}$ BAS-SR IP record the dispersed $\mathrm{x}$-rays at the position of the detecting surface. IP possess some advantages such as reasonably high spatial resolution and well- measured parameters including spectral sensitivity, image fade rate, and spatial resolution ${ }^{[31-33]}$. After exposure to x-rays, a Fuji BAS 5000 IP scanner reads out the dispersed 
Table 1. Design parameters of the Curved Crystal Spectrometers (CCS).

\begin{tabular}{lcc}
\hline & LCCS & HCCS \\
\hline Crystal & $\alpha$-quartz $(10-11) 70 \mathrm{~mm}(L) \times 12 \mathrm{~mm}(W) \times 0.27 \mathrm{~mm}(T)$ & 300 \\
Curvature radius $(\mathrm{mm})$ & 150 & $17-100$ \\
Energy range $(\mathrm{keV})$ & $10-56$ & $200-1500$ \\
Detecting distance $(\mathrm{mm})$ & $200-1500$ & $327-54$ \\
Resolution $(E / \Delta)$ & $331-57$ & Fujifilm BAS-SR IPs $74 \mathrm{~mm}(L) \times 20 \mathrm{~mm}(W)$ \\
Recording medium & \\
\hline
\end{tabular}

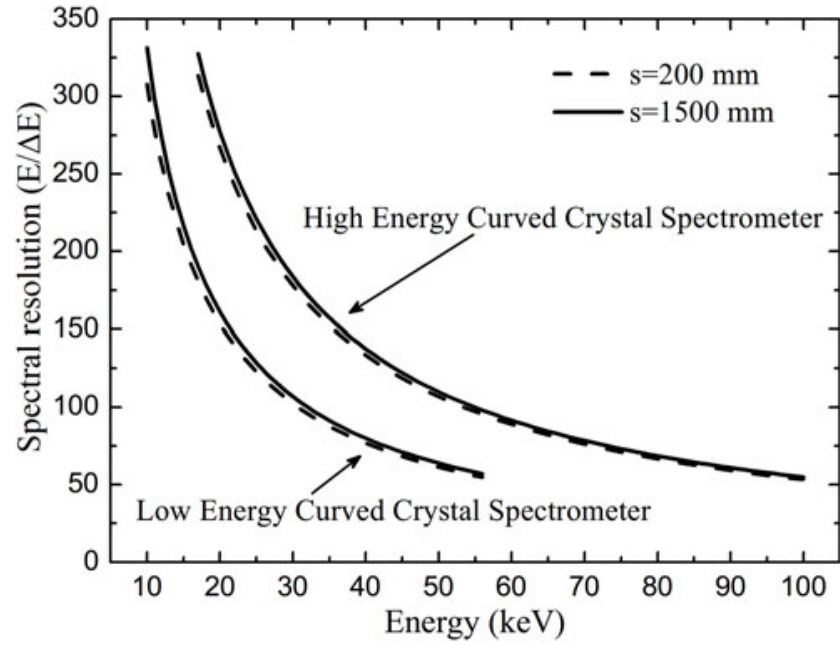

Figure 5. Spectral resolution of the LCCS and HCCS spectrometers at detecting distances of 200 and $1500 \mathrm{~mm}$. The working distance of the recording medium is fixed at $d_{0}=22 \mathrm{~mm}$.

image from the IP. The intensity of the image is expressed as a photo-stimulated luminescence (PSL) value, which is proportional to the absorbed energy in the IP's sensitive layer. The spectral sensitivity of IP describes the relationship of the PSL value and the X-ray photon energy, as shown in the insert of Figure $6^{[32]}$. Combining the IP sensitivity data from Ref. [32], and the integrated reflectivity of curved crystal calculated by the XOP code in Figure 5, we estimated the theoretical spectral sensitivities of the two spectrometers, as shown in Figure 6. The sensitivities are on the order of $10^{-8} \mathrm{PSL} /$ photon. Such low sensitivity is mainly caused by the low integral reflectivity of the curved crystal with narrow Darwin width and high-energy resolution. Ref. [34] had adopted an asymmetric curved crystal to optimize the spectral sensitivity.

\subsection{Spectral resolution of the spectrometers}

Spectral resolution $(E / \Delta E)$ is a crucial parameter for an $\mathrm{x}$ ray spectrometer. Curved crystal spectrometers can achieve high spectral resolution due to the crystal's narrow Darwin width. The actual spectral resolution of curved crystal spectrometer is determined by many factors, including the

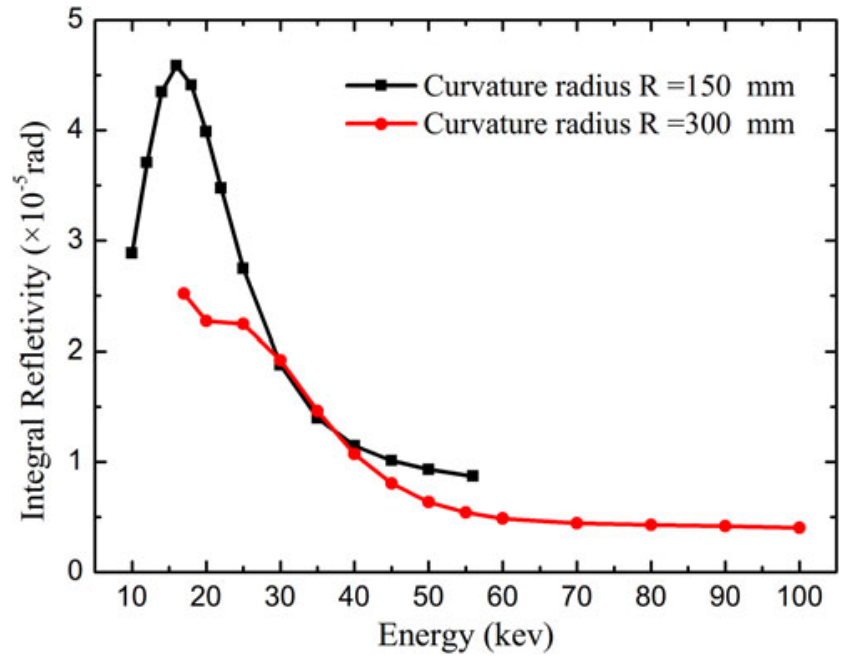

Figure 6. The energy-dependent integrated reflectivity of the curved crystals. The $\alpha$-quartz (10-11) crystals' curvature radii are $300 \mathrm{~mm}$ (HCCS) and $150 \mathrm{~mm}$ (LCCS).

Darwin width, asymmetric angle and thickness of the curved crystal, spatial resolution and working distance of the recording medium, and the position and size of the x-ray source. Seely et al. ${ }^{[35]}$ described a geometrical model to calculate the spectral resolution for the case of symmetric curved crystals. They pointed out that, for small $\mathrm{x}$-ray sources, the spectral resolution of the spectrometer is mainly determined by the spatial resolution of the recording medium for cases with small working distances (the distance between the recording medium and the Rowland circle, see Figure 1), but that the resolution was mainly determined by the lateral size of the $\mathrm{X}$-ray source for large recording medium working distances.

To illustrate the intrinsic performance of the spectrometers, we calculated the spectral resolution of the spectrometers (see Figure 4) using Seely's approach ${ }^{[35]}$ without considering the effect of the x-ray source size. The calculation parameters were: crystal thickness, $T=0.27 \mathrm{~mm}$; IP spatial resolution, $\delta d=0.11 \mathrm{~mm}^{[32]}$; recording medium (IP here) working distance, $d_{0}=22 \mathrm{~mm}$ and two different detecting distances, $s=200$ and $1500 \mathrm{~mm}$. The spectral resolution, which is not sensitive to the detecting distance, decreases with the $\mathrm{x}$-ray photon energy but increases with the curvature radius of the curved crystal. As the working 


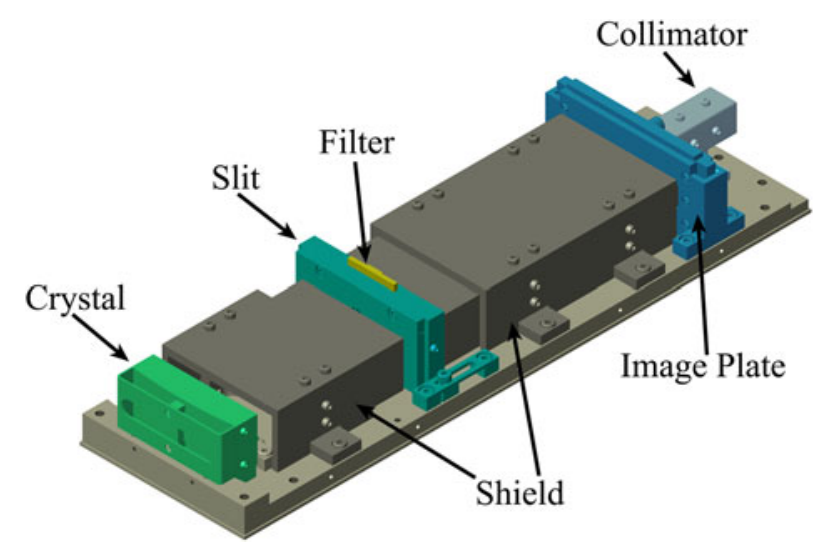

Figure 7. Schematic diagram of the HCCS spectrometer.

distance is small here, the spectral resolution is mainly affected by the spatial resolution of the recording medium. Thus, the spectral resolution can possibly be improved by increasing the working distance, which will be verified in our experiments.

\section{Mechanical design}

Mechanical modules of the HCCS, shown in Figure 7, include crystal, slit, shield, collimator, IP and lids (not shown). The LCCS spectrometer is similar to the HCCS but with different constructive parameters.

The curved crystal is the crucial module for the spectrometer. The $\alpha$-quartz crystals that we used were symmetrically cut into thin slices with the (11-20) surface plane and the (10-11) edge plane. Since thinly sliced quartz is elastic at room temperature, it can be bent without additional heating. To bend the crystal cylindrically, a pair of concave-convex cylindrical aluminum substrates were precision machined. First, we aligned the edges of the crystal slice with the predrawn lines on the convex substrate. Then, the crystal slice was pressed to cylindrical shape by the concave substrate. Pressure on the concave substrate was delivered by an elastic plastic to avoid crushing the crystal. Finally, glue was used to fix the crystal slice on the convex substrate for long-term stability.

The slit positioned at the polychromatic crossover point on the central optic axis is essential to the spectrometer. Since the diffraction efficiency of the crystal is on the order of $10^{-5}$ (see Figure 5), very weak x-rays that transmit through the crystal will contaminate the diffracted signal. A $1 \mathrm{~mm}$ width slit made in a dual layer of $3 \mathrm{~mm}$ thick tantalum and $3 \mathrm{~mm}$ lead was used to block straight-through transmitted $\mathrm{x}$ rays, combined with a shielding bar before the crystal made from $4 \mathrm{~mm}$ thick tantalum with a center collimating aperture. The transmission is less than $10^{-11}$ for $120 \mathrm{keV}$ photon energy $\mathrm{x}$-rays. Because the position of the slit changes with the detecting distance (see Figure 2), a movable slit with two guide rails was designed to make the spectrometer suitable over a broad detecting distance ranging from 200 to $1500 \mathrm{~mm}$. An additional shielding box, made from $3 \mathrm{~mm}$ thick lead, was incorporated among the crystal, slit, and IP modules to block stray $\mathrm{x}$-rays.

The IP is installed in a light-tight cassette to preserve the IP data from visible light. This module can be fixed at three different positions where the IP working distances are 7, 22, and $52 \mathrm{~mm}$. A diode laser and two collimating apertures one is on the shielding bar before the crystal, the other is placed before the IP - are used to align the spectrometer to the $\mathrm{x}$-ray source.

\section{Experimental result}

The spectrometers were tested using a Mo anode x-ray source working at $35 \mathrm{kV}$ peak voltage and $20 \mathrm{~mA}$ current. The detecting distance of the spectrometers is about $500 \mathrm{~mm}$. Spectral images recorded by the LCCS and HCCS spectrometers are shown in Figure 8. The black spots in the image centers are saturated signals from $\mathrm{x}$-rays that transmit straight through the collimating apertures (there are no filters in the collimating apertures). Over-exposure and slight misalignment make the spots much bigger than the collimating apertures and asymmetric. High-quality and symmetric Mo spectral lines were observed on both sides of the images. The detailed spectra are shown in Figure 8(c) and (d). In Figure 8(c), the flat top at the $\mathrm{K} \alpha$ line peak is caused by over-exposure, which also causes the appearance of the characteristic $\mathrm{K} \alpha$ line $(16.6 \mathrm{keV})$ of $\mathrm{Nb}$ impurities in the Mo anode. Figure 8(d) shows two clearly separated Mo $\mathrm{K} \alpha$ lines and thinner $\mathrm{K} \beta$ lines than those in Figure 8(c), indicating higher spectral resolution of HCCS. As shown in Figure 8, the energy coverage of the LCCS and HCCS spectrometers are 10-56 and 17-100 keV, respectively. So the $\mathrm{Nb} \mathrm{K} \alpha$ line did not appear in the image recorded by HCCS (Figure 8(d)).

As mentioned previously, increasing the working distance of the recording medium will induce lateral movement and separation of the spectral lines, leading to enhanced spectral resolution. On the other hand, the line width in the offRowland-circle position increases, thus decreasing the spectral resolution. However, the spectral lines separate faster than the line width increases ${ }^{[35,36]}$, thus the spectral resolution can possibly be improved by increasing the working distance. We confirmed this idea experimentally, as shown in Figure 9. The spectral images in Figure 9 correspond to IP working distances in the LCCS spectrometer of 7, 22, and $52 \mathrm{~mm}$. From left to right in Figure 9, the visible lines are $\mathrm{Mo} \mathrm{K} \beta_{2}(19.96 \mathrm{keV}), \mathrm{K} \beta_{1}(19.61 \mathrm{keV}), \mathrm{K}$ edge of the filter $\mathrm{Nb}(18.98 \mathrm{keV})$, and unseparated $\mathrm{Mo} \mathrm{K} \alpha$, respectively. Separations between the lines increase obviously with larger working distance while the line widths change little. The Mo K $\beta_{1}$ line was selected for detailed analysis of the spectral 


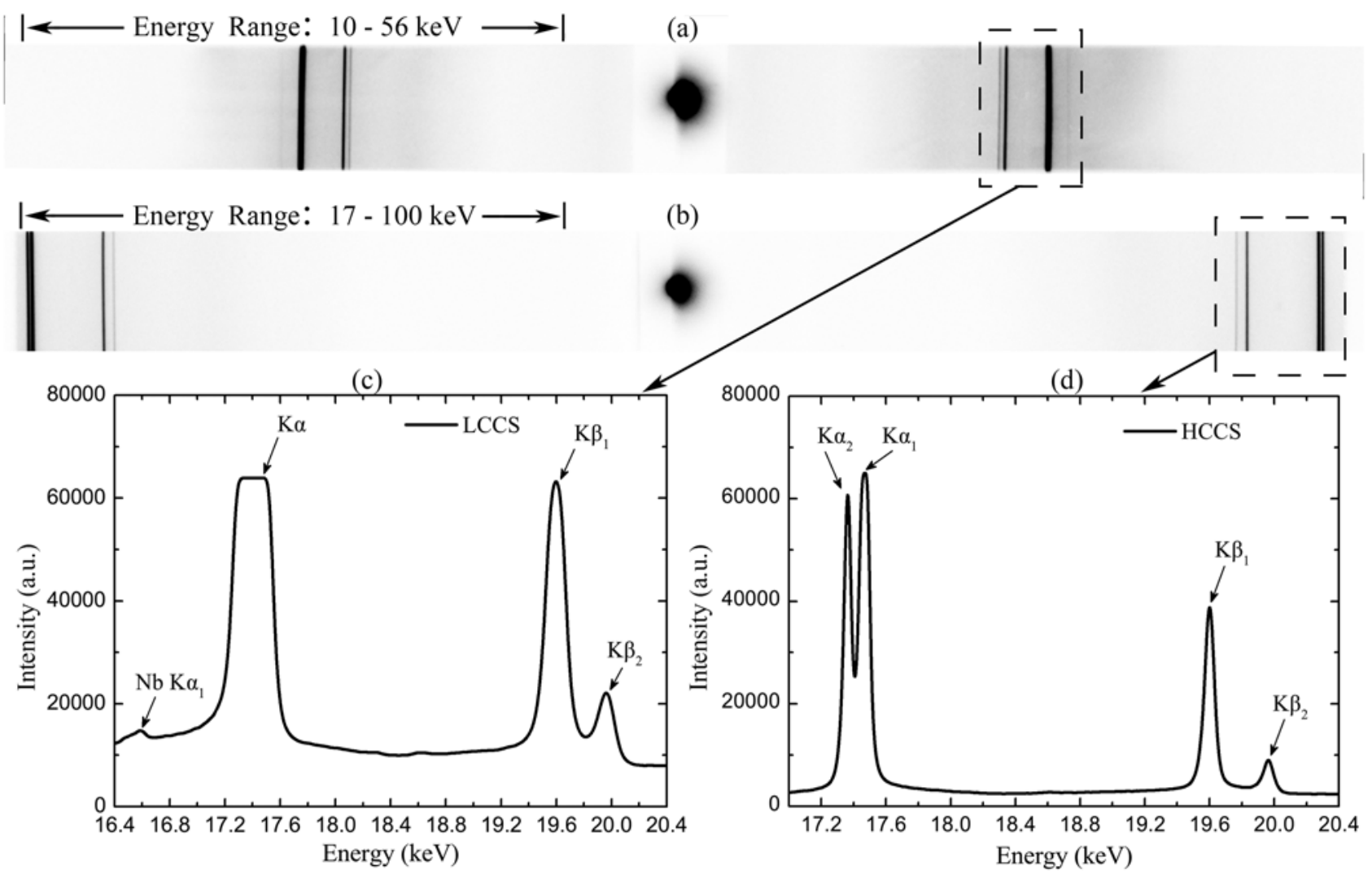

Figure 8. Experimental spectral images of a Mo anode x-ray source measured by (a) LCCS and (b) HCCS. Details of K lines are shown in (c) and (d), for LCCS and HCCS, respectively. The exposure times are $60 \mathrm{~s}$ for LCCS and $30 \mathrm{~s}$ for HCCS.

resolution $(E / \Delta E)$, because it is of moderate intensity and clearly separated from other spectral lines. The energy spread $\Delta E$ can be obtained from the full width at half maximum (FWHM) of the Gaussian fitting curve of the Mo $\mathrm{K} \beta_{1}$ line. The spectral resolutions $E / \Delta E$ are $138 \pm 4$, $140 \pm 4$, and $183 \pm 8$ for the 7,22 , and $52 \mathrm{~mm}$ working distances, respectively. The uncertainties are estimated from the Gaussian fits. The experimental results shown in Figure 10 agree well with the theoretical results using Seely's approach ${ }^{[35]}$. The parameters used in the calculation were: crystal thickness, $T=0.27 \mathrm{~mm}$; IP spatial resolution, $\delta d=0.11 \mathrm{~mm}$; detecting distance, $s=500 \mathrm{~mm}$ and lateral size of the x-ray source, $l s=1 \mathrm{~mm}$. The theoretical curve shows that the spectral resolution increases with the working distance. However, the increase of working distance will cause a loss of energy coverage.

Another test experiment was conducted at the XingGuangIII ps/PW laser facility in the Research Center of Laser Fusion in China. The laser energy delivered on the Silver nanowire array structure target ${ }^{[37]}$ was $24.8 \mathrm{~J}$ with the pulse duration of $592 \mathrm{fs}$, corresponding to a peak power of $42 \mathrm{TW}$. The HCCS was placed in the target chamber with a detecting distance of $290 \mathrm{~mm}$ and an angle of approximately $20^{\circ}$ from the normal of target back surface. Typical x-ray spectrum
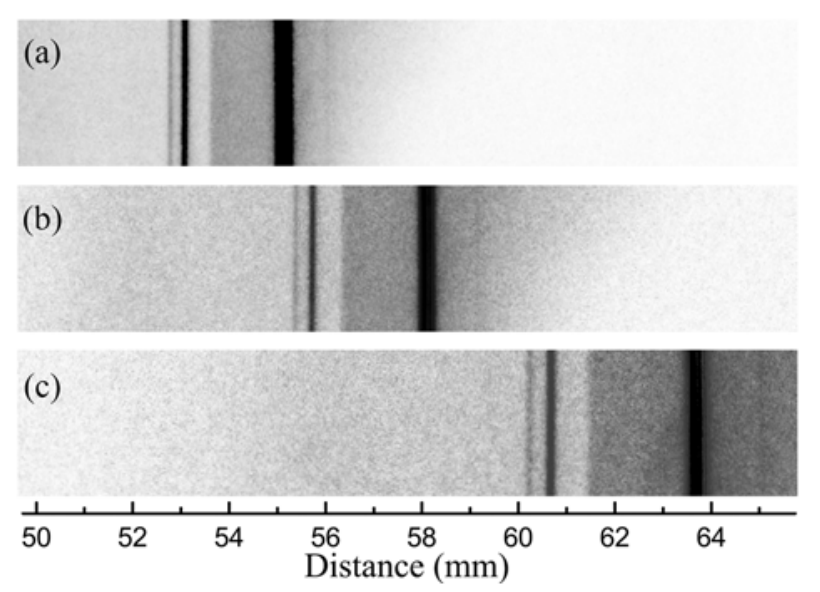

Figure 9. Spectral images of the Mo anode $x$-ray source obtained by LCCS with various working distances $d_{0}$. (a) $d_{0}=7 \mathrm{~mm}$; (b) $d_{0}=22 \mathrm{~mm}$; (c) $d_{0}=52 \mathrm{~mm}$. The abscissa represents the distance from the left side of image.

was shown in Figure 11, which provides the $\mathrm{Ag} \mathrm{K} \alpha$ and $\mathrm{K} \beta$ spectral lines clearly. However, the poor signal-to-noise ratio indicated that additional lead shielding was needed to block stray high-energy $(\mathrm{MeV}) \mathrm{x}$-rays in the short laser-solid interaction experiment. 


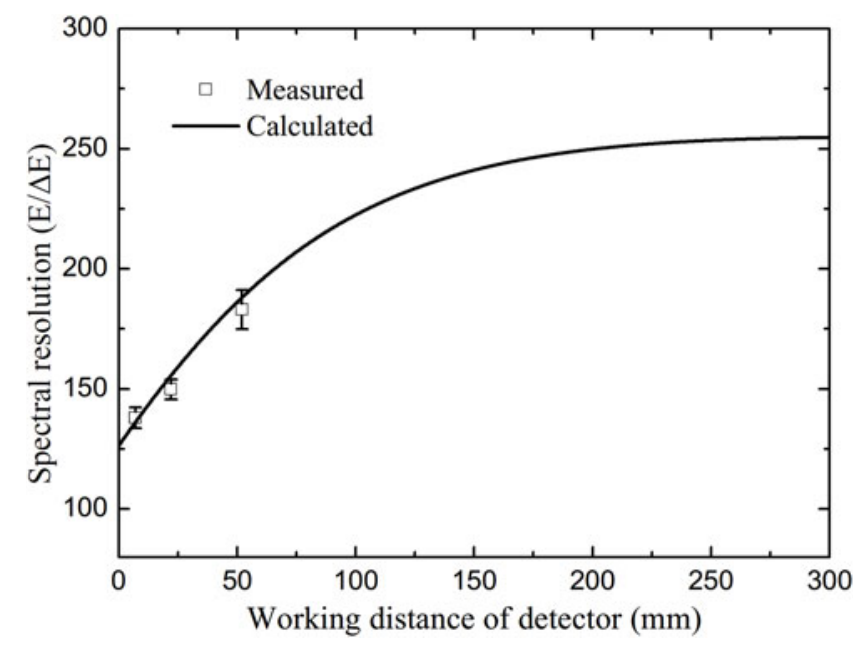

Figure 10. Experimentally measured and theoretically calculated spectral resolution at various working distances.

(a)

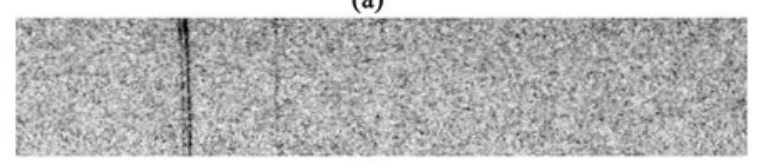

(b)

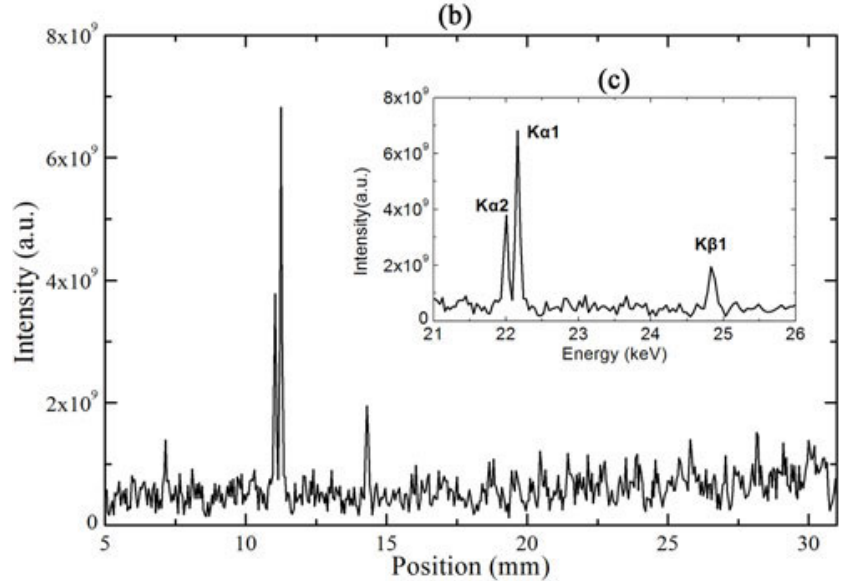

Figure 11. Ag spectral images recorded using the HCCS at the XGIII laser facility (a), the detail characteristic $\mathrm{K}$ lines of $\mathrm{Ag}(\mathrm{b}, \mathrm{c})$.

\section{Conclusion}

We designed two transmission curved crystal spectrometers to diagnose the hard $\mathrm{x}$-ray emission in the $10-100 \mathrm{keV}$ spectral range in the laser fusion experiment of Compton radiography of implosion targets at the ShenGuang-III laser facility in China. These spectrometers employ two cylindrically curved $\alpha$-quartz (10-11) crystals with curvature radii of $150 \mathrm{~mm}$ to cover the $10-56 \mathrm{keV}$ photon range, and $300 \mathrm{~mm}$ to cover the $17-100 \mathrm{keV}$ photon range. The optical design, including the sensitivity, the spectral resolution, and the mechanical design of the spectrometers, was presented. We obtained high-quality spectra of a Mo anode x-ray source. Theoretical and experimental results show that the spectral resolution can be enhanced by increasing the distance between the Rowland circle and the recording medium. In the future, we plan to add additional shielding and absolutely calibrate the spectrometers for quantitative measurement of hard x-ray spectra.

\section{Acknowledgments}

The authors are grateful to Xie Ning, Zhang Shaofeng, Yang Guohong, Wei Mingxi and Yan Ji for assistance in the experiment. This work is supported by the Natural Science Foundation of China under Grant Nos. 11105147, 11375197 and 11175179 .

\section{References}

1. T. Doppner, C. A. Thomas, L. Divol, E. L. Dewald, P. M. Celliers, D. K. Bradley, D. A. Callahan, S. N. Dixit, J. A. Harte, S. M. Glenn, S. W. Haan, N. Izumi, G. A. Kyrala, G. LaCaille, J. K. Kline, W. L. Kruer, T. Ma, A. J. MacKinnon, J. M. McNaney, N. B. Meezan, H. F. Robey, J. D. Salmonson, L. J. Suter, G. B. Zimmerman, M. J. Edwards, B. J. MacGowan, J. D. Kilkenny, J. D. Lindl, B. M. Van Wonterghem, L. J. Atherton, E. I. Moses, S. H. Glenzer, and O. L. Landen, Phys. Rev. Lett. 108, 135006 (2012).

2. T. Doppner, E. L. Dewald, L. Divol, C. A. Thomas, S. Burns, P. M. Celliers, N. Izumi, J. L. Kline, G. LaCaille, J. M. McNaney, R. R. Prasad, H. F. Robey, S. H. Glenzer, and O. L. Landen, Rev. Sci. Instrum. 83, 10E508 (2012).

3. S. P. Regan, R. Epstein, B. A. Hammel, L. J. Suter, J. Ralph, H. Scott, M. A. Barrios, D. K. Bradley, D. A. Callahan, C. Cerjan, G. W. Collins, S. N. Dixit, T. Doeppner, M. J. Edwards, D. R. Farley, S. Glenn, S. H. Glenzer, I. E. Golovkin, S. W. Haan, A. Hamza, D. G. Hicks, N. Izumi, J. D. Kilkenny, J. L. Kline, G. A. Kyrala, O. L. Landen, T. Ma, J. J. MacFarlane, R. C. Mancini, R. L. McCrory, N. B. Meezan, D. D. Meyerhofer, A. Nikroo, K. J. Peterson, T. C. Sangster, P. Springer, and R. P. J. Town, Phys. Plasmas 19, 056307 (2012).

4. S. H. Glenzer, B. J. MacGowan, P. Michel, N. B. Meezan, L. J. Suter, S. N. Dixit, J. L. Kline, G. A. Kyrala, D. K. Bradley, D. A. Callahan, E. L. Dewald, L. Divol, E. Dzenitis, M. J. Edwards, A. V. Hamza, C. A. Haynam, D. E. Hinkel, D. H. Kalantar, J. D. Kilkenny, O. L. Landen, J. D. Lindl, S. Lepape, J. D. Moody, A. Nikroo, T. Parham, M. B. Schneider, R. P. J. Town, P. Wegner, K. Widmann, P. Whitman, B. K. F. Young, B. Van Wonterghem, L. J. Atherton, and E. I. Moses, Science 327, 1228 (2010).

5. J. A. Oertel, R. Aragonez, T. Archuleta, C. Barnes, L. Casper, V. Fatherley, T. Heinrichs, R. King, D. Landers, F. Lopez, P. Sanchez, G. Sandoval, L. Schrank, P. Walsh, P. Bell, M. Brown, R. Costa, J. Holder, S. Montelongo, and N. Pederson, Rev. Sci. Instrum. 77, 10E308 (2006).

6. B. Yaakobi, R. Epstein, and F. J. Marshall, Phys. Rev. A 44, 8429 (1991).

7. R. Florido, R. C. Mancini, T. Nagayama, R. Tommasini, J. A. Delettrez, S. P. Regan, and B. Yaakobi, Phys. Rev. E 83, 066408 (2011).

8. B. R. Maddox, H. S. Park, B. A. Remington, C. Chen, S. Chen, S. T. Prisbrey, A. Comley, C. A. Back, C. Szabo, J. F. Seely, U. Feldman, L. T. Hudson, S. Seltzer, M. J. Haugh, and Z. Ali, Phys. Plasmas 18, 056709 (2011). 
9. J. F. Hansen, S. G. Glendinning, R. F. Heeter, and S. J. Brockington, Rev. Sci. Instrum. 79, 013504 (2008).

10. R. Tommasini, S. P. Hatchett, D. S. Hey, C. Iglesias, N. Izumi, J. A. Koch, O. L. Landen, A. J. MacKinnon, C. Sorce, J. A. Delettrez, V. Y. Glebov, T. C. Sangster, and C. Stoeckl, Phys. Plasmas 18, 056309 (2011).

11. B. R. Maddox, H. S. Park, B. A. Remington, and M. McKernan, Rev. Sci. Instrum. 79, 10E924 (2008).

12. J. F. Seely, G. E. Holland, L. T. Hudson, C. I. Szabo, A. Henins, H. S. Park, P. K. Patel, R. Tommasini, and J. M. Laming, High Energy Density Phys. 3, 263 (2007).

13. D. J. Johnson, Rev. Sci. Instrum. 45, 191 (1974).

14. T. Ma, N. Izumi, R. Tommasini, D. K. Bradley, P. Bell, C. J. Cerjan, S. Dixit, T. Doppner, O. Jones, J. L. Kline, G. Kyrala, O. L. Landen, S. LePape, A. J. Mackinnon, H. S. Park, P. K. Patel, R. R. Prasad, J. Ralph, S. P. Regan, V. A. Smalyuk, P. T. Springer, L. Suter, R. P. Town, S. V. Weber, and S. H. Glenzer, Rev. Sci. Instrum. 83, 10E115 (2012).

15. C. D. Chen, J. A. King, M. H. Key, K. U. Akli, F. N. Beg, H. Chen, R. R. Freeman, A. Link, A. J. Mackinnon, A. G. MacPhee, P. K. Patel, M. Porkolab, R. B. Stephens, and L. D. Van Woerkom, Rev. Sci. Instrum. 79, 10E305 (2008).

16. B. Westover, A. MacPhee, C. Chen, D. Hey, T. Ma, B. Maddox, H. S. Park, B. Remington, and F. N. Beg, Phys. Plasmas 17, 082703 (2010).

17. J. W. McDonald, R. L. Kauffman, J. R. Celeste, M. A. Rhodes, F. D. Lee, L. J. Suter, A. P. Lee, J. M. Foster, and G. Slark, Rev. Sci. Instrum. 75, 3753 (2004).

18. E. L. Dewald, C. Thomas, S. Hunter, L. Divol, N. Meezan, S. H. Glenzer, L. J. Suter, E. Bond, J. L. Kline, J. Celeste, D. Bradley, P. Bell, R. L. Kauffman, J. Kilkenny, and O. L. Landen, Rev. Sci. Instrum. 81, 10D938 (2010).

19. L. T. Hudson, A. Henins, R. D. Deslattes, J. F. Seely, G. E. Holland, R. Atkin, L. Marlin, D. D. Meyerhofer, and C. Stoeckl, Rev. Sci. Instrum. 73, 2270 (2002).

20. J. F. Seely, C. I. Szabo, U. Feldman, L. T. Hudson, A. Henins, P. Audebert, and E. Brambrink, Rev. Sci. Instrum. 81, 10E301 (2010).

21. C. I. Szabo, J. Workman, K. Flippo, U. Feldman, J. F. Seely, L. T. Hudson, and A. Henins, Rev. Sci. Instrum. 81, 10E320 (2010).

22. J. Seely, C. Back, R. Deslattes, L. Hudson, G. Holland, P. Bell, and M. Miller, Rev. Sci. Instrum. 72, 2562 (2001).
23. J. Y. Mao, L. M. Chen, L. T. Hudson, J. F. Seely, L. Zhang, Y. Q. Sun, X. X. Lin, and J. Zhang, Rev. Sci. Instrum. 83, 043104 (2012).

24. Z. Zhang, H. Nishimura, T. Namimoto, S. Fujioka, Y. Arikawa, M. Nishikino, T. Kawachi, A. Sagisaka, H. Hosoda, S. Orimo, K. Ogura, A. Pirozhkov, A. Yogo, Y. Okano, H. Kiriyama, K. Kondo, S. Ohshima, and H. Azechi, Rev. Sci. Instrum. 83, 053502 (2012).

25. D. B. Sinars, D. F. Wenger, S. A. Pikuz, B. Jones, M. Geissel, S. B. Hansen, C. A. Coverdale, D. J. Ampleford, M. E. Cuneo, L. A. McPherson, and G. A. Rochau, Rev. Sci. Instrum. 82, 063113 (2011).

26. Y. Cauchois, J. Phys. 3, 203 (1932).

27. L. T. Hudson, R. D. Deslattes, A. Henins, C. T. Chantler, E. G. Kessler, and J. E. Schweppe, Med. Phys. 23, 1659 (1996).

28. S. Takagi, J. Phys. Soc. Japan 26, 1239 (1969).

29. M. Sanchez del Rio, C. Ferrero, and V. Mocella, Proc. SPIE 3151, 312 (1997).

30. E. Erola, V. Etelaniemi, P. Suortti, P. Pattison, and W. Thomlinson, J. Appl. Crystallogr. 23, 35 (1990).

31. A. L. Meadowcroft, C. D. Bentley, and E. N. Stott, Rev. Sci. Instrum. 79, 113102 (2008).

32. B. R. Maddox, H. S. Park, B. A. Remington, N. Izumi, S. Chen, C. Chen, G. Kimminau, Z. Ali, M. J. Haugh, and Q. Ma, Rev. Sci. Instrum. 82, 023111 (2011).

33. J. F. Seely, G. E. Holland, L. T. Hudson, and A. Henins, Appl. Opt. 47, 5753 (2008).

34. H. F. Beyer, D. Attia, D. Banas, E. O. Le Bigot, F. Bosch, J. C. Dousse, E. Förster, A. Gumberidze, S. Hagmann, S. Heß, J. Hoszowska, P. Indelicato, P. Jagodzinski, C. Kozhuharov, T. Krings, D. Liesen, X. Ma, B. Manil, I. Mohos, M. Pajek, D. Protić, R. Reuschl, J. Rzadkiewicz, A. Simionovici, U. Spillmann, Z. Stachura, T. Stöhlker, M. Trassinelli, S. Trotsenko, A. Warczak, O. Wehrhan, and E. Ziegler, Spectrochim. Acta B 64, 736 (2009).

35. J. F. Seely, L. T. Hudson, G. E. Holland, and A. Henins, Appl. Opt. 47, 2767 (2008).

36. F. Qian, L. Cao, S. Xiao, W. Zhou, Z. Zhao, Y. Gu, Y. Yan, and and L. Wei, High Power Laser Part. Beams 25, 2292 (2013).

37. J. Wang, Z. Zhao, W. He, B. Zhu, K. Dong, Y. Wu, T. Zhang, G. Niu, K. Zhou, N. Xie, W. Zhou, and Y. Gu, Chin. Opt. Lett. 13, 031001 (2015). 